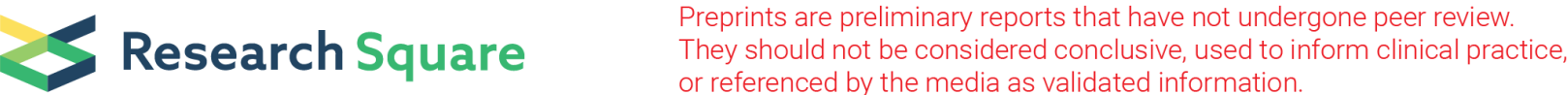

\section{The effect of polyethersulfone and nylon 6 micromembrane filters on the pyraclostrobin detection囚Adsorption performance and mechanism}

\section{Xiaolan Shao}

Hunan Agricultural University

Lejun Liu

Hunan Agricultural University

Hui Li

North Carolina A\&T: North Carolina Agricultural and Technical State University

Yue Luo

Hunan Agricultural University

Jingyu Zhao

Hunan Agricultural University

Shuai Liu

Hunan Agricultural University

Bei Yan

Hunan Agricultural University

\section{Dan Wang}

Hunan Agricultural University

Kun Luo

Hunan Agricultural University

Min Liu

Hunan Agricultural University

Lianyang Bai

Hunan Agricultural University

Xiaoyun Li

Shaanxi Normal University

Kailin Liu ( $\sim$ kailin@hunau.net )

Hunan Agricultural University https://orcid.org/0000-0002-4830-3137

\section{Research Article}

Keywords: Pyraclostrobin, Polyethersulfone micromembrane, Nylon 6 micromembrane, Adsorption mechanism 
Posted Date: March 3rd, 2022

DOI: https://doi.org/10.21203/rs.3.rs-1364050/v1

License: (c) (i) This work is licensed under a Creative Commons Attribution 4.0 International License. Read Full License 


\section{Abstract}

Adsorption of test substances on micromembrane filters during sample pretreatment before qualitative and quantitative analysis has greatly affected the accuracy of the measurement. In the present study, it was found that the adsorption rate of pyraclostrobin reached $77.7-100 \%$ when water samples of pyraclostrobin $(1 \mathrm{~mL})$ were filtered with polyethersulfone (PES) and nylon 6 filters. Therefore, the adsorption mechanisms were investigated from the kinetics, isotherms, and thermodynamics of the pyraclostrobin adsorption process, combined with Attenuated Total Reflection-Fourier Transform InfraRed (ATR-FTIR) spectroscopy and X-ray Photoelectron Spectroscopy (XPS) analysis. The adsorption of pyraclostrobin on the PES micromembrane is chemical adsorption; while Nylon 6 is physical adsorption. However, pyraclostrobin adsorption onto surface sites of Nylon 6 micromembrane was mostly through physical monolayer. The $\pi-\pi$ electron-donor-acceptor (EDA) between pyraclostrobin and PES may promote the adsorption of PES to pyraclostrobin, and hydrogen bonding between pyraclostrobin and Nylon 6 micromembrane may be involved in the adsorption. Our study also proved the addition of methanol and iodine solution was an effective strategy to reduce the adsorption effects and to increase the accuracy of the detection.

\section{Introduction}

With the development of analysis technologies, modern quantitative trace analyzing instruments such as high-performance liquid chromatography (HPLC) and high-performance liquid chromatography-tandem mass spectrometry (LC-MS-MS), which has the advantages of high sensitivity and simple operation procedures, can directly and effectively detect and quantify the tested substances in aqueous samples after filtration. However, the adsorption of the test substances on the micromembrane during filtration has greatly affected the accuracy of the analysis. Therefore, it is necessary to study the adsorption law and mechanisms to determine the extent of these adsorption effects and to figure out appropriate strategies to minimize these effects.

In agricultural production, pesticides are widely used and play a significant role in controlling diseases and pests and regulating plant growth (Guo et al., 2007). Once applied, however, pesticides would enter the surrounding environment through pesticide drift, surface water flow, and soil deposition (Druart et al., 2011). Pyraclostrobin, a strobilurin fungicide, has been applied to a wide range of crops due to its high activity against fungi (Li et al., 2018c)and its unique function to improve crop growth characteristics (Li et al., 2020). Consequently, it has been frequently detected in water bodies and sediments adjacent to highly agricultural areas. For example, the maximum concentration of pyraclostrobin detected in Yarra Valley in Australia was $0.1 \mu \mathrm{g} / \mathrm{L}$ and at higher concentration $(0.28 \mu \mathrm{g} / \mathrm{L})$ in wetlands in the Rainwater Basin of Nebraska close to the row-crop farmland (Mimbs IV et al., 2016). Moreover, pyraclostrobin can adversely affect non-targeted aquatic organisms and aquatic microbial systems due to the toxicity of itself and its intermediates. Increasing number of studies has reported its great negative impact on aquatic organisms (Li et al., 2018a), amphibian, Juvenile snails (Fidder et al., 2016), mice (Tuttle et al., 2019) and aquatic microorganism ecology (Lu et al., 2019) In addition, human exposure to pyraclostrobin may also induce 
health hazards, such as eye injury and skin irritation (Control and Prevention, 2008). Therefore, determining concentration levels of the environmental occurrence of pyraclostrobin is very essential.

Various analytical techniques have been applied to determine concentration levels of pyraclostrobin in various matrices. Most of these analytical methods require a filtration step during sample preparation. However, many studies have evidenced the adsorption behaviors of small organic molecules onto different organic polymers, which will affect the recovery rate and determination accuracy of these analyzes. For instance, PES micromembrane has a strong adsorption effect on some pesticides and estrogen (Morin et al., 2018). Taking this advantage, PES hollow fibers have been used to extract several polarities from caffeine (Prieto et al., 2012) and polar chlorinated pesticides from water (Prieto et al., 2014). Lately, PES tubes have been successfully used as a passive sampler for more hydrophobic compounds in water and emerging micropollutants in seawater (Mijangos et al., 2018). Similarly, Nylon 6 micromembrane also has a strong adsorption effect on some organic matter (Han et al., 2010). In addition, the adsorption phenomenon between Nylons and several organics (e.g. ethinylestradiol, humic acid, fulvic acid and bisphenol A) (Jasni et al., 2017; Tang et al., 2021)and pesticides (e.g. diazinon, diuron profenofo and promertryn) (Wei et al., 2015) have been reported. The adsorption behavior of three typical strobilurins (azoxystrobin, picoxystrobin, and pyraclostrobin) on polystyrene and polyethylene has also been investigated (Hai et al., 2020).

The physicochemical properties of the adsorbent rely on van der Waals forces, the physical properties, hydrophobicity, and surface functional groups of the adsorbent and the adsorbate, which can significantly affect the adsorption process and efficiency. Yang et al. demonstrated a strong linear relationship between the adsorption amount of a given organic substance, such as phenanthrene, and the specific surface area of carbon nanomaterials (Yang and Xing, 2010) Van der Waals forces are widely present interaction forces in adsorption phenomena (Liu et al., 2020). In addition, the hydrophobic interactions have also been shown to significantly affect the adsorption capacity of adsorbents for adsorbed organic matter substances (Guo et al., 2019; Liu et al., 2020). A covalent bond compound, which consists of hydrogen and an atom with a large electronegativity and a small radius (e.g. O, F, and $\mathrm{N})$, often complexes with adsorbent via hydrogen bonds. For instance, the - $\mathrm{COOH},-\mathrm{OH}$ (Han et al., 2010; Han et al., 2012), and - $\mathrm{NH}_{2}$ groups of organic chemicals can complex via hydrogen-bonding (Alnajrani and Alsager, 2020; Han et al., 2010; Li et al., 2018b; Liu et al., 2020). The $\pi-\pi^{\star}$ interaction has also played an important role in the adsorption process between adsorbent and adsorbate molecules (Sun et al., 2020). Moreover, studies have shown that the effect of electrostatic interaction on the adsorption of ionizable organic compounds cannot be ignored (Alnajrani and Alsager, 2020; Liu et al., 2020; Yang and Xing, 2010).

However, the adsorption behaviors of pyraclostrobin on micromembranes and to what extent these micromembranes will affect the accuracy of determination of environmental samples are still not fully understood. In addition, micromembranes of different surface structures may induce different interaction rate and/or mechanisms with pyraclostrobin. Therefore, Nylon 6 and PES-two commonly used filter membrane materials, which have been widely used as syringe filters for pretreatment of laboratory 
samples (Boparai et al., 2011) and medical infusion, ascribed to their excellent characteristics and low prices, are selected as model micromembranes. The objectives of the present study are 1) to investigate the law of pyraclostrobin adsorption on PES and Nylon 6 micromembranes during filtration; 2) to study and clarify the adsorption mechanisms of pyraclostrobin on these two micromembranes based on the kinetics, isotherms, and thermodynamics of adsorption, combining with attenuated total reflectionFourier transform infrared (ATR-FTIR) spectroscopy and X-ray photoelectron spectroscopy (XPS) analysis, and 3) to explore the influence of methanol addition on pyraclostrobin adsorption on the micromembrane filters and to find out methods to eliminate the adsorption effect.

\section{Materials And Methods}

\subsection{Chemicals and materials}

The physicochemical properties of pyraclostrobin (Jiangsu Heng long Plant Protection Co., Ltd) were presented in (Table S1). The physicochemical properties of the two micromembranes (Tianjin Branch billion Lung Experimental Equipment CO., Ltd) were presented in (Table S2) and (Fig. S1). Methanol (Sigma, Germany), acetonitrile (Sigma, Germany), and sodium azide (Hefei Bomei Biotechnology Co., Ltd, China) were analytical grades.

\subsection{Adsorption of pyraclostrobin on micromembrane filters at different concentration levels}

The adsorption law of filtration on two microfilters were explored at 0.5 and $1.0 \mathrm{mg} / \mathrm{L}$ concentration levels. Since the nominal capacity of the microfilter was $10 \mathrm{~mL}$, we studied the adsorption of pyraclostrobin within 1-10 mL of solution volume on PES and Nylon6 microfilters. A disposable medical syringe was used to aspirate the aqueous solution of pyraclostrobin, which was filtered through a new filter until the cumulative filtration volume reached $10 \mathrm{~mL}$. Each syringe was rinsed with $10 \mathrm{~mL}$ pyraclostrobin solution at the experimental concentration before use. Each milliliter filtrate was placed in a $2 \mathrm{~mL}$ brown sample vial, individually, and another $1 \mathrm{~mL}$ solution was directly injected into the sample bottle without filtration as a comparison treatment. Concentrations of filtered pyraclostrobin were directly determined on HPLC (Agilent1260, Agilent, America) right after sample collection. The effect of methanol and iodine on filtration adsorption was explored following the same processes.

\subsection{Adsorption kinetics of pyraclostrobin on micromembranes}

Each $50 \mathrm{~mm}$ diameter micromembrane was cut into a sheet-shaped micromembrane with a diameter of 2 $\mathrm{mm}$ using a punch to facilitate weighing. Adsorption Adsorption experiments were performed in the dark for $24 \mathrm{~h}$ using a $50 \mathrm{~mL}$ Erlenmeyer flask in a thermostatic shaker $(220 \mathrm{rpm}, 298.15 \mathrm{~K}$ ) in batch experiments. The effect of PES micromembrane dosage on the adsorption process was investigated by applying different amounts of PES micromembranes $(0.125$ to $0.625 \mathrm{~g} / \mathrm{L})$ in solutions with an initial pyraclostrobin concentration of $1 \mathrm{mg} / \mathrm{L}$. Similarly, the effect of Nylon 6 micromembrane dosage on the 
adsorption process was investigated by applying Nylon 6 micromembranes ( 1 to $6 \mathrm{~g} / \mathrm{L}$ ) in $1 \mathrm{mg} / \mathrm{L}$ pyraclostrobin solutions.

Experiments for adsorption kinetics were conducted in solutions with an initial pyraclostrobin concentration of $1 \mathrm{mg} / \mathrm{L}$. The adsorbent: solution ratios of PES micromembrane adsorption experiment were 0.01: 40 (g: $\mathrm{mL})$, and 0.03: $30(\mathrm{~g}: \mathrm{mL})$ for Nylon 6 micromembrane. Concentrations of pyraclostrobin after adsorption were analyzed at different time intervals from 0 to $28 \mathrm{~h}$. Experiments for the adsorption isotherms were carried out at concentrations of pyraclostrobin ranging from 0.3 to $1.6 \mathrm{mg} / \mathrm{L}$ at $298.15 \mathrm{~K}$. The adsorption thermodynamics experiment was conducted ranging from 288.15 to $308.15 \mathrm{~K}$. All experiments were conducted in three replicates.

All samples were extracted according to the extraction experiment method in the Supplementary Materials (Text S1) all experiments were repeated in triplicate and detected by HPLC. The statistical analysis for adsorption kinetics, isotherms, and thermodynamics are described in Supplementary Materials (Text S2).

\subsection{Quantitative analysis of pyraclostrobin using HPLC}

HPLC was utilized to quantify pyraclostrobin concentrations. The chromatographic column is a Thermo $\mathrm{C} 18$ column $(150 \times 4.6 \mathrm{~mm}, 5 \mu \mathrm{m})$, maintained at $28^{\circ} \mathrm{C}$. The mobile phase, consisted of methanol, acetonitrile, and water (35: $45: 20, \mathrm{~V}: \mathrm{V}: \mathrm{V})$, was set at a flow rate of $0.8 \mathrm{~mL} / \mathrm{min}$. The injection volume of the tested solution was $20 \mu \mathrm{L}$. The detection wavelength for pyraclostrobin is $275 \mathrm{~nm}$, the detection limit of HPLC was $0.05 \mathrm{mg} / \mathrm{L}$.

\subsection{Mercury pressure testing, ATR-FTIR and XPS of micromembrane}

The porosity, total pore area, and pore size distribution of micromembrane with a pore size of $0.22 \mu \mathrm{m}$ were tested by Auto Pore IV 9500 mercury porosimeter. To reach adsorption saturation, the volume ratio between the mass of Nylon 6/PES micromembrane and the $1.5 \mathrm{mg} / \mathrm{L}$ pyraclostrobin solution was 0.04 : 500 (g: $\mathrm{mL}$ ). A blank control group with ultrapure water as the background solution was conducted in parallel. After adsorption for $24 \mathrm{~h}$, the micromembrane was freeze-dried for nearly $24 \mathrm{~h}$. Then, the ATRFTIR (Nicolet iS50 from Thermo Fisher Scientific, USA) and XPS (Thermo ESCOLAB 250Xi, USA) was used to analyze the surface chemical elements, structure, and groups of the micromembrane before and after adsorption of pyraclostrobin.

\section{Results And Discussion}

\subsection{Adsorption of pyraclostrobin by micromembrane filters}

The adsorption rates of different concentrations of pyraclostrobin on PES and Nylon 6 micromembranes after consecutive constant-volume filtering are shown in (Fig. $1(\mathbf{a}, \mathbf{b})$ ). Size exclusion is the main principle of micromembrane, which means that filters can effectively remove particles larger than their 
pore size. However, the molecular size of most organic compounds classified as pesticides is close to 1 $\mathrm{nm}$ (Plakas and Karabelas, 2012), which is much smaller than the pore size of micromembrane $(0.22 \mu \mathrm{m}$ pore size), indicating that the adsorption resulted in the concentration decreased.

The adsorption rate of pyraclostrobin on PES micromembrane is greatly higher than that on Nylon 6 micromembrane at both 0.5 and $1.5 \mathrm{mg} / \mathrm{L}$ concentration levels. For example, when the filtration volume reaches the 6 th $\mathrm{mL}$, the adsorption rate of pyraclostrobin to PES is $66.3 \%$, and its adsorption rate on Nylon 6 filters is only $7.50 \%$, using $0.5 \mathrm{mg} / \mathrm{L}$ of pyraclostrobin solution as feed solution. The main cause for this dramatic difference could be the difference in the total pore area (PESロNylon 6) of these two micromembranes (Fig. S1). In addition, specific functional groups on the surface of the filter membranes that may bind to the filter material can be another important cause for this phenomenon (Han et al., 2012). The effect of concentrations of feed solution on the adsorption rate is also obvious that the higher the concentration, the less the volume of the feed solution required for the adsorption process to reach saturation. This phenomenon might be related to the adsorption capacity of the microfiltration membrane (Wei et al., 2015).

\subsection{Adsorption kinetics of pyraclostrobin on PES and Nylon6 micromembranes}

Several adsorption kinetic models have been used to evaluate the performance of the adsorbent and to investigate the adsorption mass transfer mechanisms (Fig. 1 (c, d)). Pyraclostrobin adsorption on both micromembranes increases rapidly within $0-2 \mathrm{~h}$. The adsorption of pyraclostrobin on Nylon 6 micromembrane reaches equilibrium in the next 3 hours. Whereas, the increasing rate of pyraclostrobin adsorption capacity on PES micromembrane slows down within 2-24 $\mathrm{h}$. The adsorption process achieves equilibrium at $24 \mathrm{~h}$. Therefore, $24 \mathrm{~h}$ of reaction is selected in the following experiments to ensure adsorption equilibrium. Stability experiment was used to ensure the indicated pyraclostrobin was stable under the relevant experimental conditions within the experimental time range (Fig. S2).

As shown in (Fig. 1) and (Table 1), the equilibrium adsorption capacity of PES micromembrane for pyraclostrobin is about 9 times that of Nylon6 micromembrane. The coefficient of determination $\left(R^{2}\right)$ of

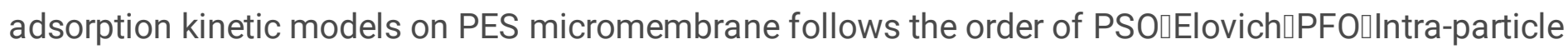
diffusion, among which PSO shows the smallest chi-square $\left(\chi^{2}\right)$ value. The $\mathrm{R}^{2}$ of the adsorption models

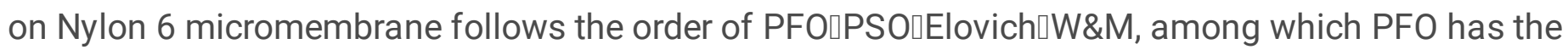
smallest $\chi^{2}$ value. Therefore, the PSO model better explains the adsorption kinetics of pyraclostrobin on PES micromembrane and PFO better describes that on Nylon 6 micromembrane. This probably indicates that there are fewer active adsorption sites on Nylon 6 micromembrane, but more abundant adsorption sites on PES micromembrane (Guo and Wang, 2019). Furthermore, this also suggests that the external or internal diffusion could be the rate-controlling step in the adsorption process on Nylon 6 micromembrane. Besides the adsorption onto surface sites, mass transfer and intraparticle diffusion may also involve in the adsorption process on PES and micromembrane . 
Table 1

Parameters of different kinetic models for pyraclostrobin sorption to PES and Nylon 6 micromembranes (at $298.13 \mathrm{~K}, \mathrm{pH}=6.92$ ).

\begin{tabular}{|c|c|c|c|c|}
\hline Model & Formula & Paramters & PES & Nylon 6 \\
\hline \multirow[t]{4}{*}{ PFO } & \multirow[t]{4}{*}{$Q_{t}=q_{e}\left(1-e^{-k_{1} t}\right)$} & $q_{e}$ & 2.72 & 0.31 \\
\hline & & $k_{1}$ & 0.67 & 1.22 \\
\hline & & $R_{1}^{2}$ & 0.90 & 0.98 \\
\hline & & $x^{2}$ & 5.37 & 0.64 \\
\hline \multirow[t]{4}{*}{ PSO } & \multirow{4}{*}{$Q_{t}=\frac{k_{2} q_{e}^{2} t}{1+k_{2} q_{e} t}$} & $q_{e}$ & 2.84 & 0.34 \\
\hline & & $k_{2}$ & 0.44 & 6.19 \\
\hline & & $R_{2}^{2}$ & 0.98 & 0.92 \\
\hline & & $x^{2}$ & 0.65 & 2.93 \\
\hline \multirow[t]{4}{*}{ Elovich Model } & \multirow{4}{*}{$Q_{t}=\frac{1}{b} \ln (A B)+\frac{1}{b} \ln (t)$} & a & 195.73 & 52.78 \\
\hline & & b & 3.60 & 34.27 \\
\hline & & $R_{e}^{2}$ & 0.88 & 0.66 \\
\hline & & $x^{2}$ & 5.38 & 12.87 \\
\hline \multicolumn{5}{|c|}{$\begin{array}{l}\text { Notes: } k_{1}(1 / \mathrm{min}) \text { an } k_{2}(\mathrm{~g} / \mathrm{mg} / \mathrm{min}) \text { are the pseudo-first-order and pseudo-second-order kinetic } \\
\text { constants; } q_{e}(\mathrm{mg} / \mathrm{g}) \text { is the adsorption equilibrium; } Q_{t} \text { is the unit adsorption capacity of } \\
\text { micromembrane at any time }(\mathrm{mg} / \mathrm{g}) \text {, and } \mathrm{t} \text { is the adsorption time }(\mathrm{h}) ; \mathrm{A}(\mathrm{mg} / \mathrm{g} / \mathrm{min}) \text { is the initial } \\
\text { adsorption rate constant, B }(\mathrm{mg} / \mathrm{g}) \text { represents the coverage rate and activation energy value of the } \\
\text { activated adsorption site. }\end{array}$} \\
\hline
\end{tabular}

According to the W\&M model, when $\mathrm{Q}_{\mathrm{t}}$ versus $\mathrm{t}^{0.5}$ is multi-linear, the adsorption process involves two or more steps (Taqvi et al., 2007). The fitting results of the W\&M model show that the adsorption process of pyraclostrobin on the two microporous membranes could be divided into three stages (as shown in Fig. 1 $(\mathbf{e}, \mathbf{f}))$. The initial steeper part represents the diffusion of the surface on a micromembrane, the second linear steeper represents the gradual adsorption phase, where the diffusion within the particles or pores are the rate-limiting step, and the third steeper is the final equilibrium phase. Since this graph does not pass through the origin, intra-particle diffusion is not the only rate-limiting step. Pyraclostrobin molecules in the solution are first transferred from the aqueous phase to the surface of the micromembrane and then diffused into the interior of the microporous membrane through the pore filling (Wu et al., 2009). In the second part, the value of intercept $\mathrm{C}$ is related to the thickness of the boundary layer (Kavitha and Namasivayam, 2007). A larger intercept indicates that the surface diffusion has a greater role in the rate- 
limiting step. Therefore, the much greater intercept value of the PES adsorption process suggests a more greater role of surface diffusion than that of Nylon 6.

\subsection{Adsorption isotherm of pyraclostrabin on micromembranes}

To find a suitable adsorbent/solution ratio, at which the adsorption rate is above $20 \%$ (preferably $>50 \%$ ) (Boesten, 1994), and to explore the influence of micromembrane dosages on adsorption capacity and adsorption rate, different amounts of micromembranes were applied in pyraclostrobin solutions with an initial concentration of $1 \mathrm{mg} / \mathrm{L}$. As shown in (Fig. S2), the adsorption rate of pyraclostrobin on two kinds of micromembranes increases dramatically with the growth of their dosages. Whereas the adsorptive capacity of different micromembranes decreases sharply with the growth of micromembrane dosages. The increase of pyraclostrobin adsorption rate may ascribe to more adsorption sites that are provided by increased dosages of micromembranes. However, the adsorption capacity decreases, most likely due to the reduction in aqueous solution concentration (Li et al., 2021) and the competition between pyraclostrobin molecules for available adsorption sites (Wang et al., 2020). Taking the above-discussed factors and the method detection limit $(0.05 \mathrm{mg} / \mathrm{L})$ into consideration, the dosages of PES and Nylon 6 micromembranes at $0.25 \mathrm{~g} / \mathrm{L}$ and $2.0 \mathrm{~g} / \mathrm{L}$, respectively, were applied for further adsorption experiments in this study.

Four isotherm models have been applied to study the adsorption mechanisms and mass transfer during the adsorption process of pyraclostrobin on micromembranes. As shown in (Fig. 1g) and (Table 2), the Freundlich model is the best fit for the adsorption process on the PES micromembrane. This indicates that the adsorption process may follow the multi-layer asorption on heterogeneous surfaces (Li et al., 2021; Zaheer et al., 2019). The value of the constant $n$ in the Freundlich model is 1.758 1 , which indicates physical adsorption. As shown in (Fig. 1h) and (Table 2), the linear model is the best fit for adsorption isotherms on Nylon 6 micromembrane. The linear model suggests a low coverage rate of the adsorbate on the active sites of the adsorbent by deriving the equation, which further suggests monolayer adsorption of pyraclostrobin on Nylon 6 micromembrane at the experimental concentration (Wang and Guo, 2020). In addition, the Langmuir model is the worst fit, indicating that pyraclostrobin adsorption on micromembranes is not likely to be molecular adsorption on homogeneous surfaces (Li et al., 2021). 
Table 2

Parameters of different isotherm models for pyraclostrobin adsorption on PES and Nylon 6 micromembranes (at $298.13 \mathrm{~K}, \mathrm{pH}=6.92$ ).

\begin{tabular}{|c|c|c|c|c|}
\hline Model & Equation & Parameter & PES & Nylon 6 \\
\hline \multirow[t]{3}{*}{ Linear isotherm } & \multirow[t]{3}{*}{$Q_{e}=K_{L} C_{e}$} & $K_{L}$ & 4.343 & 0.475 \\
\hline & & $R_{L}^{2}$ & 0.949 & 0.975 \\
\hline & & P's r & 0.974 & 0.981 \\
\hline \multirow[t]{4}{*}{ Freundlich isotherm } & \multirow{4}{*}{$Q_{e}=K_{F} C_{e^{\frac{1}{n}}}$} & $K_{F}$ & 3.089 & 0.411 \\
\hline & & $n$ & 1.758 & 1.509 \\
\hline & & $R_{F}^{2}$ & 0.982 & 0.977 \\
\hline & & $x^{2}$ & 0.975 & 1.353 \\
\hline \multirow[t]{4}{*}{ Langmuir isotherm } & \multirow{4}{*}{$Q_{e}=\frac{q_{m} K_{l} C_{e}}{1+K_{l} C_{e}}$} & $K_{l}$ & 2.508 & 1.179 \\
\hline & & $q_{m}$ & 3.793 & 0.724 \\
\hline & & $R_{l}^{2}$ & 0.966 & 0.961 \\
\hline & & $\chi^{2}$ & 11.957 & 2.324 \\
\hline \multirow[t]{4}{*}{ Temkin isotherm } & \multirow[t]{4}{*}{$Q_{e}={ }_{\bar{B}}^{R T} \ln \left(A C_{e}\right)$} & $B$ & 2978.446 & 17716.838 \\
\hline & & A & 24.447 & 14.441 \\
\hline & & $R_{T}^{2}$ & 0.960 & 0.942 \\
\hline & & $x^{2}$ & 13.776 & 3.476 \\
\hline \multicolumn{5}{|c|}{$\begin{array}{l}\text { Notes: } Q_{e}(\mathrm{mg} / \mathrm{g}) \text { and } C_{e}(\mathrm{mg} / \mathrm{L}) \text { respectively represent the adsorption mass of the adsorb rate and its } \\
\text { concentration in the solution; } K_{L}(\mathrm{~L} / \mathrm{g}) \text { and } K_{F}(\mathrm{~L} / \mathrm{g}) \text { are the partition coefficient; } K_{l}(\mathrm{~L} / \mathrm{mg}) \text { is the ratio } \\
\text { of adsorption rate to the desorption rate; } q_{m} \text { is the adsorption capacity calculated from the Langmuir } \\
\text { model; } \mathrm{R}(8.314 \mathrm{~J} / \mathrm{mol} \cdot \mathrm{K}) \text { is the gas molar constant, } \mathrm{T}(\mathrm{K}) \text { is the absolute temperature, } \mathrm{A}(\mathrm{L} / \mathrm{g}) \text { and } \mathrm{B} \\
(\mathrm{J} / \mathrm{mol}) \text { are both a constant. }\end{array}$} \\
\hline
\end{tabular}

These results are consistent with the above kinetic results. Generally speaking, hydrophobic compounds are more easily adsorbed via hydrophobic polymerization (Fang et al., 2019; Wang et al., 2020). Pyraclostrobin has no $p K_{\mathrm{a}}$, and its octanol-water partition coefficient ( $\left.\log K_{\mathrm{ow}}\right)$ is 3.99 (Table S1). Studies have found that the order of the adsorption capacity of polystyrene and polyethylene microplastics for three typical strobilurins (pyraclostrobin, picoxystrobin and azoxystrobin) is consistent with their Log $K_{\text {ow }}$ (Hai et al., 2020). Therefore, hydrophobic interaction is more conducive to the distribution of 
pyraclostrobin on the hydrophobic PES micromembrane. Nylon 6 is a hydrophilic organic polymer with abundant amide functional groups. The $-\mathrm{NH}$ of amide functional groups can form a hydrogen bond $(\mathrm{NH} \cdots \mathrm{O}=\mathrm{C})$ with the carbonyl group from pyraclostrobin (Han et al., 2010; Han et al., 2012). In addition, the $-\mathrm{NH}$ of amide functional groups can also form hydrogen bond ( $\mathrm{NH} \cdots \pi$ hydrogen bond) with $\pi-$ electron cloud of pyraclostrobin aromatic groups that act as the acceptor (An et al., 2009). The $\pi-\pi^{*}$ interaction may be formed by the phenyl groups of the adsorbent and the adsorbate, one as electron donor and the other as electron acceptor (Archin et al., 2019; Sun et al., 2020). Peng et al. studied the adsorption of antibiotics on graphene and biochar and verified via the density functional theory that as the number of aromatic rings increases, the adsorption capacity increases significantly, indicating the importance of $\pi-\pi^{\star}$ interaction in the adsorption process (Peng et al., 2016). Therefore, the adsorption of pyraclostrobin on PES micromembrane might be majorly via $\pi-\pi^{\star}$ interaction, due to the existence of phenyl in their structures.

\subsection{Adsorption thermodynamics of pyraclostrabin on micromembranes}

To study the thermodynamics of pyraclostrobin adsorption, adsorption isotherms at different temperatures were investigated at the concentration range within $0.4-1.2 \mathrm{mg} / \mathrm{L}$. As shown in (Fig. S3),

(Fig. S4) and (Table S4), the linear model is the best fit for adsorption isotherms on both micromembranes. Interestingly, this result is slightly different from the adsorption isotherms at the concentration range of $0.3-1.6 \mathrm{mg} / \mathrm{L}$ at $298.15 \mathrm{~K}$. After refitting the data $(0.3-1.3 \mathrm{mg} / \mathrm{L})$ for the PES micromembrane in the section 3.3, the linear model is the best fitting model, which is consistent with the results here. Therefore, we suggest that the adsorption of pyraclostrobin on PES micromembrane is monolayer adsorption at low concentrations due to abundant adsorption sites on the micromembrane.

By plotting InK against 1/T (Fig. S5), the thermodynamic parameters of adsorption, enthalpy changes $(\Delta \mathrm{H})$ can be obtained from the slope of the correlation line and entropy changes $(\Delta S)$ from the intercept. All Gibbs Free Energy $(\Delta G)$ is negative (Table S6), indicating a decrease in the driving force of adsorption due to highly occupied energy adsorption sites. Thus adsorption processes on both micromembranes are spontaneous and thermodynamically stable. Generally, the changes in the value of enthalpy $(\Delta \mathrm{H})$ for physical adsorption is less than $20 \mathrm{~kJ} / \mathrm{mol}$, which is smaller than that of chemisorption (80 to 200 $\mathrm{kJ} / \mathrm{mol}$ ). In this study, the absolute value of all enthalpy changes is within the range of $0-20 \mathrm{~kJ} / \mathrm{mol}$, which indicates the dominancy of physical adsorption during the adsorption process of pyraclostrobin on these micromembranes (Archin et al., 2019).

The decrease in the negative value of $\triangle \mathrm{G}$ of PES micromembrane with an increase in temperature indicates that pyraclostrobin adsorption on PES micromembrane becomes more favorable at higher temperature. The positive values of $\Delta \mathrm{H}$ suggest the pyraclostrobin adsorption onto PES micromembrane to be endothermic. This means that the temperature increase is beneficial to the adsorption of pyraclostrobin on the PES micromembrane. The positive $\Delta S$ demonstrates increased randomness of pyraclostrobin molecules at the solid/solution interface during the adsorption on the PES 
micromembrane. Although the entropy value of the pyraclostrobin molecule adsorbed to the PES micromembrane is reduced, the temperature has a greater influence on the entropy value of the entire system; therefore, the entropy value changes to a positive value.

The negative values of $\Delta \mathrm{H}$ indicate that the adsorption process of pyraclostrobin onto Nylon 6 micromembrane is exothermic. It also implies that the increase in temperature is not conducive to the progress of the adsorption process. The negative value of $\Delta S$ means that the freedom of movement of the pyraclostrobin molecules decreases, so as the chaos of the entire system after the adsorption reaction occurs. Because the pyraclostrobin molecule changes from free movement in the solution before adsorption to two-dimensional movement on the micromembrane after adsorption.

\subsection{ATR-FTIR and XPS analysis used to probe adsorption mechanisms}

To study the effect of specific surface area on adsorption, we used a mercury pressure meter to measure the total pore area of PES and Nylon 6 micromembranes. As shown in (Fig. S1), the pore size of PES micromembrane is mainly distributed between 95.45 and $676.45 \mathrm{~nm}$, while the pore size of Nylon 6 micromembrane is mainly distributed within $95.37-1619.30 \mathrm{~nm}$ and $32.40-50.34 \mathrm{~nm}$. The total pore areas of PES and Nylon 6 micromembrane are 10.77 and $4.875 \mathrm{~m}^{2} / \mathrm{g}$ (Table S2), respectively. The total pore area is proportional to the adsorption capacity, which is consistent with our previous speculation. But the difference in total pore area could not sufficiently explain their difference in adsorption capacity. This suggests other factors that affect the adsorption capacity.

ATR-FTIR was used to further study the adsorption mechanisms, specifically the interaction bonds between pyraclostrobin and polymeric membranes. The strong band at $1716 \mathrm{~cm}^{-1}$ is the $-\mathrm{C}=0$ stretching vibration peak associated with the benzene ring (Fig. S6). The four larger peaks at 1599, 1548, 1505, and $1479 \mathrm{~cm}^{-1}$ represent the benzene ring skeleton stretching vibration. Peaks at 1439,1255 , and $1107 \mathrm{~cm}^{-1}$ indicate the occurrence of $-\mathrm{N}-\mathrm{C}=\mathrm{O}$ bond absorption, $\mathrm{N}-\mathrm{C}$ stretching vibration and $-\mathrm{C}-\mathrm{O}$ bond stretching vibration, respectively. The anti-symmetric sulfone-based $\left(-\mathrm{SO}_{2-}\right)$ stretching vibration absorption is supported by the peaks at $1299 \mathrm{~cm}^{-1}$ and $1323 \mathrm{~cm}^{-1}$, and the peak at $1153 \mathrm{~cm}^{-1}$ is evidence for symmetrical sulfone-based stretching vibration. The characteristic proofs of the anti-symmetric stretching vibration absorption of aromatic ether show up at $1241 \mathrm{~cm}^{-1}$ (Fig. S6). also demonstrated the imido $(-\mathrm{NH})$ in the amide group stretching vibration at $3300 \mathrm{~cm}^{-1}$, and its bending vibration at $1542 \mathrm{~cm}^{-}$ 1 . The carbonyl group (CO-) in the amide group stretching vibration is shown at $1637 \mathrm{~cm}^{-1}$.

There is no significant difference in the infrared spectra of the two micromembranes before and after adsorption, which indicates that PES and Nylon 6 has no or negligible chemical interaction with pyraclostrobin, other than obvious physical adsorption. Besides, the absence of detectable pyraclostrobin may be due to the following reasons: $i$ ) the infrared spectrum is not sensitive to the small amount of adsorbed pyraclostrobin (Alnajrani and Alsager, 2020; Li et al., 2021); ii) multi-aperture PES micromembrane has a shielding effect on pyraclostrobin signal (Alnajrani and Alsager, 2020). 
At the same time, XPS spectral analysis of micromembrane showed the presence of $\mathrm{C}$ - $\mathrm{Cl}$ after the adsorption, indicating the adsorption of pyraclostrobin on the PES micromembrane (Fig. 2b and Table S6). The N-H and C-N in CKP treatment may be due to impurity contamination in the test process or material production process (Fig. S7). The presence of $\pi-\pi^{*}$ shake-up satellite peak is found in 0.04P and CKP treatments, which can provide good conditions for the formation of $\pi-\pi^{\star}$ bonds between pyraclostrobin and PES molecules. Besides, $\mathrm{PES}$ has an electronegative group $\mathrm{O}=\mathrm{S}=\mathrm{O}$, which is similar to sulfamethoxazole, that can act as the $\pi$ electron acceptor (Ahmed et al., 2017). In addition, the presence of a lone pair orbital formally localized at the adjacent carbonyl bond and benzene ring $\mathrm{N}$ atom with nature of pure p-type and a low electron occupancy (1.75e) allows an electron-donating capacity in pyraclostrobin (Dominguez et al., 2021). Thus, the $\pi-\pi$ electron-donor-acceptor (EDA) can increase the adsorption of pyraclostrobin to PES, which is supported by the binding energy of $\mathrm{O}=\mathrm{S}=\mathrm{O}$ increase (Fig. 2), and the adsorption of the PES micromembrane filter to pyraclostrobin reduced in the iodine solution than that in the water (Fig. 4a) because the iodine may eliminate $\pi-\pi$ interaction (Deng et al., 2011).

The presence of $\mathrm{C}-\mathrm{Cl}$ and $\mathrm{C}-\mathrm{O}$ after the adsorption indicates the adsorption of pyraclostrobin on Nylon 6 micromembrane (Fig. 2 (d, e)) and Table S7). Dominguez et al. found that $\mathrm{C}=0$ in the pyraclostrobin molecule is the most negative (Dominguez et al., 2021). Therefore, pyraclostrobin and Nylon 6 form $\mathrm{N}$ $\mathrm{H} \cdots \mathrm{O}=\mathrm{C}$ hydrogen bonds (Han et al., 2010), where the electrons are attracted by oxygen atoms, resulting in a decrease in the electron density of $\mathrm{C}-\mathrm{N}$ and an increase in the electron density of $\mathrm{O}=\mathrm{C}$, and an increase in the $\mathrm{C}-\mathrm{N}$ binding energy and the decrease of $\mathrm{O}=\mathrm{C}$ binding energy.

The forms of possible hydrogen bonds of PES, Nylon 6 and pyraclostrobin are shown in (Fig. 3). First, The carbonyl group of pyraclostrobin can form a $\pi$ hydrogen bond with the hydrogen on the benzene ring $(\mathrm{CH} \cdots \mathrm{O}=\mathrm{C}$ hydrogen bond) (Fig. 3a). Second, the $\mathrm{NH}$ of the amide group could also form a hydrogen bond with the oxygen atom of the carbonyl group in the pyraclostrobin molecules ( $\mathrm{NH} \cdots \mathrm{O}$ hydrogen bond). Additionally, The $\mathrm{NH}$ of the amide group can be formed aromatic hydrogen bond ( $\mathrm{NH} \cdots$ Hydrogen bond) with the $\pi$ electron cloud of the pyraclostrobin aromatic group acting as acceptor (Fig. 3b).

\subsection{Effects of methanol and iodine on the adsorption of pyraclostrobin on micromembrane filters}

As the proportion of methanol increases, the membrane adsorption effect gradually decreases until it was eliminated and the elimination effect of Nylon 6 micromembrane is more obvious than the PES (Fig. 4). When $60 \%$ of methanol was added to the aqueous sample, the adsorption effect of pyraclostrobin on these two kinds of micromembranes was almost eliminated. The presence of methanol might increase the solubility of pyraclostrobin and improve the wettability of hydrophobic surfaces of PES micromembrane (Wei et al., 2015) The adsorption rate of pyraclostrobin on PES micromembrane reduced in the $2.0 \mathrm{mmol} / \mathrm{L}$ iodine solution than that in the water (Fig. 4a), which might be ascribed to the eliminated $\pi-\pi$ interaction by iodine (Deng et al., 2011). Though at the same time, the dissolved potassium iodide may promote $\pi-\pi$ interaction, hydrogen bonding and hydrophobicity, and increase the adsorption. But the inhibition of iodine on $\pi-\pi$ is the main effect. Whereas, the adsorption rate on Nylon 6 
micromembrane increased in the iodine solution, compared with that in water (Fig. 4b). For Nylon 6 micromembrane, the adsorption is mainly via hydrogen bond. Therefore, iodine has limited inhibiting effect on its adsorption. While dissolved potassium iodide may promote hydrogen bond and hydrophobicity. Thus, iodine promotes the adsorption of pyraclostrobin on Nylon 6 (Goh et al., 2021). Future research will be needed to explore more ways to reduce the effect of adsorption.

\section{Conclusions}

Appropriate determination of pyroclostrobin is essential to understanding its fate and transport in the environment. However, pyraclostrobin is easily adsorbed by commonly used micromembranes PES and Nylon 6 during filtration, which is a necessary step during sample pretreatment before analysis. Thus, the precision of the analysis is strongly influenced by the adsorption process. Hydrophilic micromembrane Nylon 6 can reach adsorption equilibrium more quickly than PES. The adsorption process of pyraclostrobin on PES micromembrane is better defined by the Freundlich isotherm and pseudo secondorder kinetic models, indicating that besides the multilayer adsorption onto surface sites, mass transfer and intraparticle diffusion also involve in the adsorption process. The adsorption process of pyraclostrobin on Nylon 6 micromembrane is better described by the linear isotherm and pseudo firstorder kinetic models, indicating dominant monolayer adsorption, which is mainly controlled by the physical adsorption. The adsorption of PES and Nylon 6 is spontaneous, and the pyraclostrobin adsorption on Nylon 6 is exothermic and endothermic on the PES micromembrane. Furthermore, ATRFTIR and XPS results verify the adsorption of pyraclostrobin on micromembrane to be a major physical process. In addition to the hydrophobic interforce and van der Waals interforce, the hydrogen bonding is also involved in the adsorption process on Nylon 6 micromembrane; whereas the $\pi-\pi$ EDA interaction may involve the adsorption process on PES micromembrane. Moreover, this study demonstrates an effective strategy to eliminate the adsorption effect of the micromembrane using methanol and iodine. Future investigations are needed to improve the properties of micromembranes so that to efficiently decrease the adsorption effects.

\section{Declarations}

\section{Funding}

This work was supported by the Scientific Research Project of Education Department of Hunan Province (19A231), the Science and Technology Plan Projects of Hunan Province (2020SK2033), the Hunan Province Postgraduate Teaching Platform Project (Xiangjiaotong[2019]No. 370), the Scientific-Innovative of Hunan Agricultural Sciences and Technology (2019LS05 and 2019TD03), and the Natural Science Foundation of Hunan Province (2019JJ50270).

\section{Competing Interests}

"The authors have no relevant financial or non-financial interests to disclose." 


\section{Author Contributions}

"All authors contributed to the study conception and design. Material preparation, data collection and analysis were performed by [Lejun Liu], [Hui Li] and [Kailin Liu]. Software, Computational, Conceptualization and Writing- Reviewing and Editing were performed by [Yue Luo], [Jingyu Zhao], [Shuai Liu], [Bei Yan], [Dan Wang], [Kun Luo], [Min Liu], [Lianyang Bai] and [Xiaoyun Li]. The first draft of the manuscript was written by [Xiaolan Shao] and all authors commented on previous versions of the manuscript. All authors read and approved the final manuscript."

\section{Ethical Approval}

The authors warrant that the manuscript is not submitted to multiple journals for simultaneous consideration. Submissions are original and not published elsewhere in any form or language (in part or in whole). The results are clear, honest and free from fabrication, falsification or inappropriate data processing (including image-based processing). All authors follow discipline-specific rules for acquiring, selecting and processing data.

\section{Consent to Participate}

With the consent of all authors, hereby assign to Enviromental Science and Pollution Research, the copyright in the above identified article to be transferred, including supplemental tables, illustrations or other information submitted in all forms and media throughout the world, in all languages and format, effective when and if the article is accepted for publication.

Authors also agree to the following terms:

A. The article submitted is not subject to any prior claim or agreement and is not under consideration for publication elsewhere.

B. The article contains no libelous or other unlawful statements and does not contain any materials that violate proprietary right of any other person, company, organization, and nation.

C. Ifthe article was prepared jointly with other authors, the author(s) agree with the authorship sequence.

\section{Availability of data and materials}

All authors ensure that all data and materials, as well as software applications or custom code, support the claims they make and meet field standards.

\section{References}

1. Ahmed MB et al (2017) Single and competitive sorption properties and mechanism of functionalized biochar for removing sulfonamide antibiotics from water. Chem Eng J 311:348-358 
2. Alnajrani MN, Alsager OA (2020) Removal of antibiotics from water by polymer of intrinsic microporosity: Isotherms, kinetics, thermodynamics, and adsorption mechanism. Sci Rep 10:1-14

3. An F et al (2009) Adsorption mechanism and property of a novel adsorption material PAM/SiO2 towards 2, 4, 6-trinitrotoluene. J Hazard Mater 168:352-357

4. Archin S et al (2019) Optimization and modeling of simultaneous ultrasound-assisted adsorption of binary dyes using activated carbon from tobacco residues: response surface methodology. J Clean Prod 239:118136

5. Boesten J (1994) Influence of soil/liquid ratio on the experimental error of sorption coefficients in relation to OECD guideline 106. Proceedings of 5th international workshop on environmental behaviour of pesticides and regulatory aspects, Brussels, pp. 26-29

6. Boparai HK et al (2011) Kinetics and thermodynamics of cadmium ion removal by adsorption onto nano zerovalent iron particles. J Hazard Mater 186:458-465

7. Control CfD, Prevention (2008) Acute pesticide poisoning associated with pyraclostrobin fungicidelowa, 2007. MMWR: Morbidity and mortality weekly report. 56, 1343-1345

8. Deng $Y$ et al (2011) Investigation of aggregation and assembly of alkali lignin using iodine as a probe. Biomacromolecules 12:1116-1125

9. Dominguez AN et al (2021) Experimental and theoretical vibrational study of the fungicide pyraclostrobin. Spectrochimica Acta Part A: Molecular and Biomolecular Spectroscopy 259:119888

10. Druart $C$ et al (2011) Snails as indicators of pesticide drift, deposit, transfer and effects in the vineyard. Sci total Environ 409:4280-4288

11. Fang $S$ et al (2019) Adsorption behavior of three triazole fungicides on polystyrene microplastics. Sci Total Environ 691:1119-1126

12. Fidder BN et al (2016) Energetic endpoints provide early indicators of life history effects in a freshwater gastropod exposed to the fungicide, pyraclostrobin. Environ Pollution 211:183-190

13. Goh JY et al (2021) High salinity enhances the adsorption of 17a-ethinyl estradiol by polyethersulfone membrane: isotherm modelling and molecular simulation.International Journal of Environmental Science and Technology.1-10

14. Guo X et al (2019) Sorption of sulfamethazine onto different types of microplastics: A combined experimental and molecular dynamics simulation study. Mar pollution Bull 145:547-554

15. Guo X, Wang J (2019) Comparison of linearization methods for modeling the Langmuir adsorption isotherm. J Mol Liquids 296:111850

16. Guo X et al (2007) The importance of fungicides/bactericides in American agriculture. World pesticides 9:21-25

17. Hai N et al (2020) Effects of Microplastics on the Adsorption and Bioavailability of Three Strobilurin Fungicides. ACS omega 5:30679-30686

18. Han J et al (2010) Adsorption of estrone in microfiltration membrane filters. Chem Eng J 165:819826 
19. Han J et al (2012) Removal of ethinylestradiol (EE2) from water via adsorption on aliphatic polyamides. Water Res 46:5715-5724

20. Jasni MJF et al (2017) Electrospun nylon 6, 6 membrane as a reusable nano-adsorbent for bisphenol A removal: Adsorption performance and mechanism. J colloid interface Sci 508:591-602

21. Kavitha D, Namasivayam C (2007) Experimental and kinetic studies on methylene blue adsorption by coir pith carbon. Bioresour Technol 98:14-21

22. Li H et al (2018a) Developmental toxicity, oxidative stress and immunotoxicity induced by three strobilurins (pyraclostrobin, trifloxystrobin and picoxystrobin) in zebrafish embryos. Chemosphere 207:781-790

23. Li H et al (2021) Adsorption of three pesticides on polyethylene microplastics in aqueous solutions: Kinetics, isotherms, thermodynamics, and molecular dynamics simulation. Chemosphere 264:128556

24. Li J et al (2018b) Adsorption of antibiotics on microplastics. Environ Pollution 237:460-467

25. Li M et al (2018c) Preparation and application of pyraclostrobin microcapsule formulations. Colloids and Surfaces A: Physicochemical and Engineering Aspects 553:578-585

26. Li P et al (2020) Evaluation of Pyraclostrobin as an Ingredient for Soybean Seed Treatment by Analyzing its Accumulation-Dissipation Kinetics, Plant-Growth Activation, and Protection Against Phytophthora sojae. J Agricultural Food Chem 68:11928-11938

27. Liu Z et al (2020) Adsorption of chlorophenols on polyethylene terephthalate microplastics from aqueous environments: Kinetics, mechanisms and influencing factors. Environ Pollution 265:114926

28. Lu T et al (2019) Ecotoxicological effects of fungicides azoxystrobin and pyraclostrobin on freshwater aquatic bacterial communities. Bull Environ Contam Toxicol 103:683-688

29. Mijangos L et al (2018) Evaluation of polar organic chemical integrative and hollow fibre samplers for the determination of a wide variety of organic polar compounds in seawater. Talanta 185:469476

30. Mimbs IV et al (2016) Occurrence of current-use fungicides and bifenthrin in Rainwater Basin wetlands. Chemosphere 159:275-281

31. Morin NA et al (2018) Kinetic accumulation processes and models for 43 micropollutants in “pharmaceutical” POCIS. Sci total Environ 615:197-207

32. Peng B et al (2016) Adsorption of antibiotics on graphene and biochar in aqueous solutions induced by $\pi-\pi$ interactions. Sci Rep 6:1-10

33. Plakas KV, Karabelas AJ (2012) Removal of pesticides from water by NF and RO membranes-a review. Desalination 287:255-265

34. Prieto A et al (2014) Evaluation of polyethersulfone performance for the microextraction of polar chlorinated herbicides from environmental water samples. Talanta 122:264-271

35. Prieto A et al (2012) Evaluation of low-cost disposable polymeric materials for sorptive extraction of organic pollutants in water samples. Anal Chim acta 716:119-127 
36. Sun Z et al (2020) Fast adsorption of BPA with high capacity based on $\pi-\pi$ electron donor-acceptor and hydrophobicity mechanism using an in-situ sp2 C dominant N-doped carbon. Chem Eng $\mathrm{J}$ 381:122510

37. Tang S et al (2021) Adsorption of fulvic acid onto polyamide 6 microplastics: Influencing factors, kinetics modeling, site energy distribution and interaction mechanisms. Chemosphere 272:129638

38. Taqvi SIH et al (2007) Sorption profile of Cd (II) ions onto beach sand from aqueous solutions. J Hazard Mater 141:37-44

39. Tuttle AH et al (2019) Choice of vehicle affects pyraclostrobin toxicity in mice. Chemosphere 218:501-506

40. Wang J, Guo X (2020) Adsorption isotherm models: Classification, physical meaning, application and solving method. Chemosphere 258:127279

41. Wang $T$ et al (2020) Adsorption behavior and mechanism of five pesticides on microplastics from agricultural polyethylene films. Chemosphere 244:125491

42. Wei L et al (2015) Effects of Microfiltration Membrane Adsorption on Detection of Pesticides in Water by Direct Injection Liquid Chromatography-Tandem Mass Spectrometry.Chinese Journal of Analytical Chemistry.1761-1765

43. Wu F-C et al (2009) Initial behavior of intraparticle diffusion model used in the description of adsorption kinetics. Chem Eng J 153:1-8

44. Yang K, Xing B (2010) Adsorption of organic compounds by carbon nanomaterials in aqueous phase: Polanyi theory and its application. Chem reviews 110:5989-6008

45. Zaheer Z et al (2019) Adsorption of methyl red on biogenic Ag@ Fe nanocomposite adsorbent: Isotherms, kinetics and mechanisms. J Mol Liquids 283:287-298

\section{Figures}



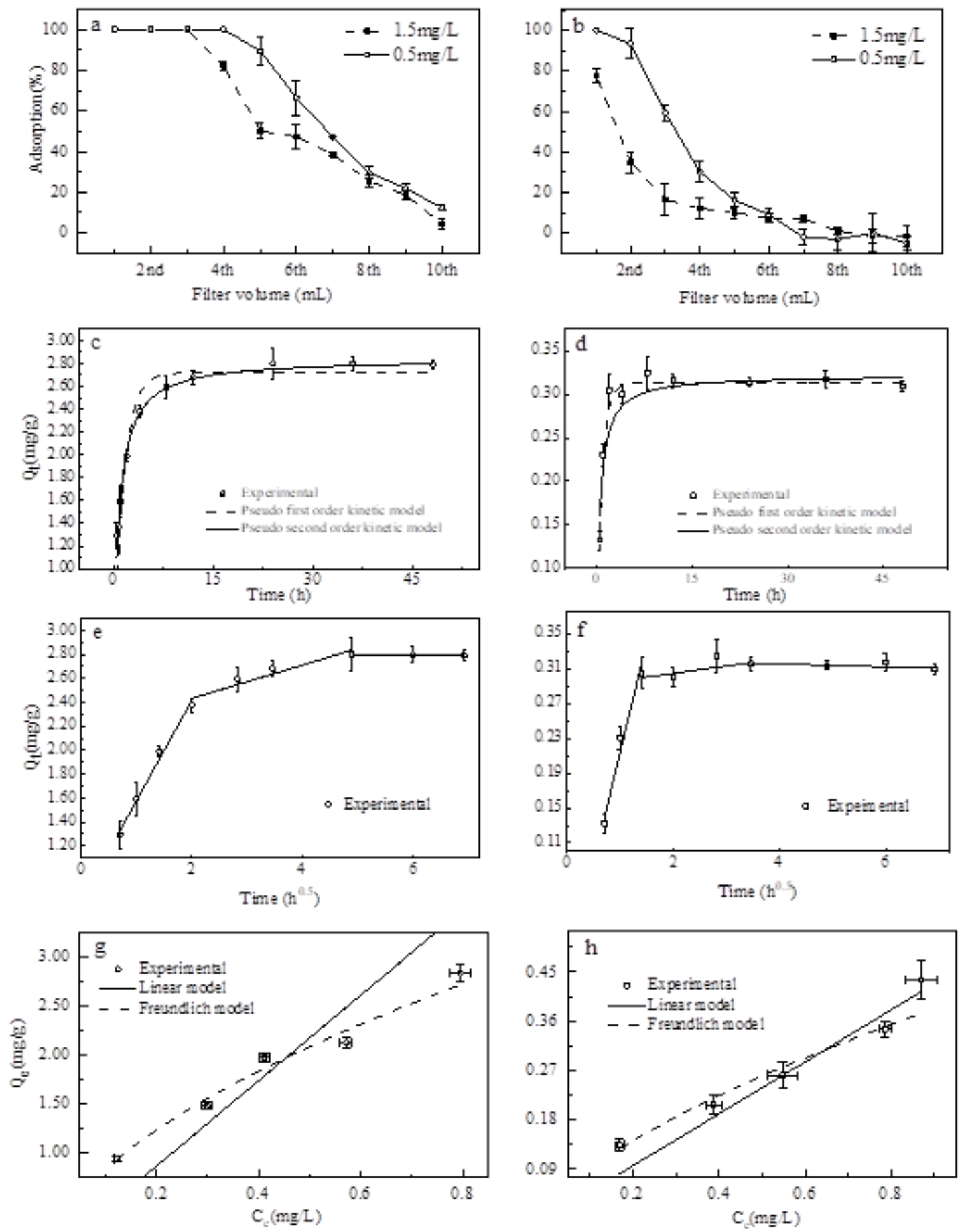

Figure 1

Adsorption rates of pyraclostrobin on PES (a) and Nylon 6 (b) micromembrane filters in the process of continuous filtration. Adsorption kinetics of pyraclostrobin to PES and Nylon 6 micromembranes. (c) and (d) are adsorption kinetics based on the pseudo-first-order and pseudo-second-order, and (e) and (f) are intraparticle diffusion models, respectively. Adsorption isotherms of pyraclostrobin on PES (g) and Nylon 6 (h) micromembranes. (initial concentration of pyraclostrobin is $1.0 \mathrm{mg} / \mathrm{L}$, adsorption time $=30 \mathrm{~h}$ and temperature $=298.13 \mathrm{~K}$, error bars represent standard deviation from the mean $(n=3)$ ). 


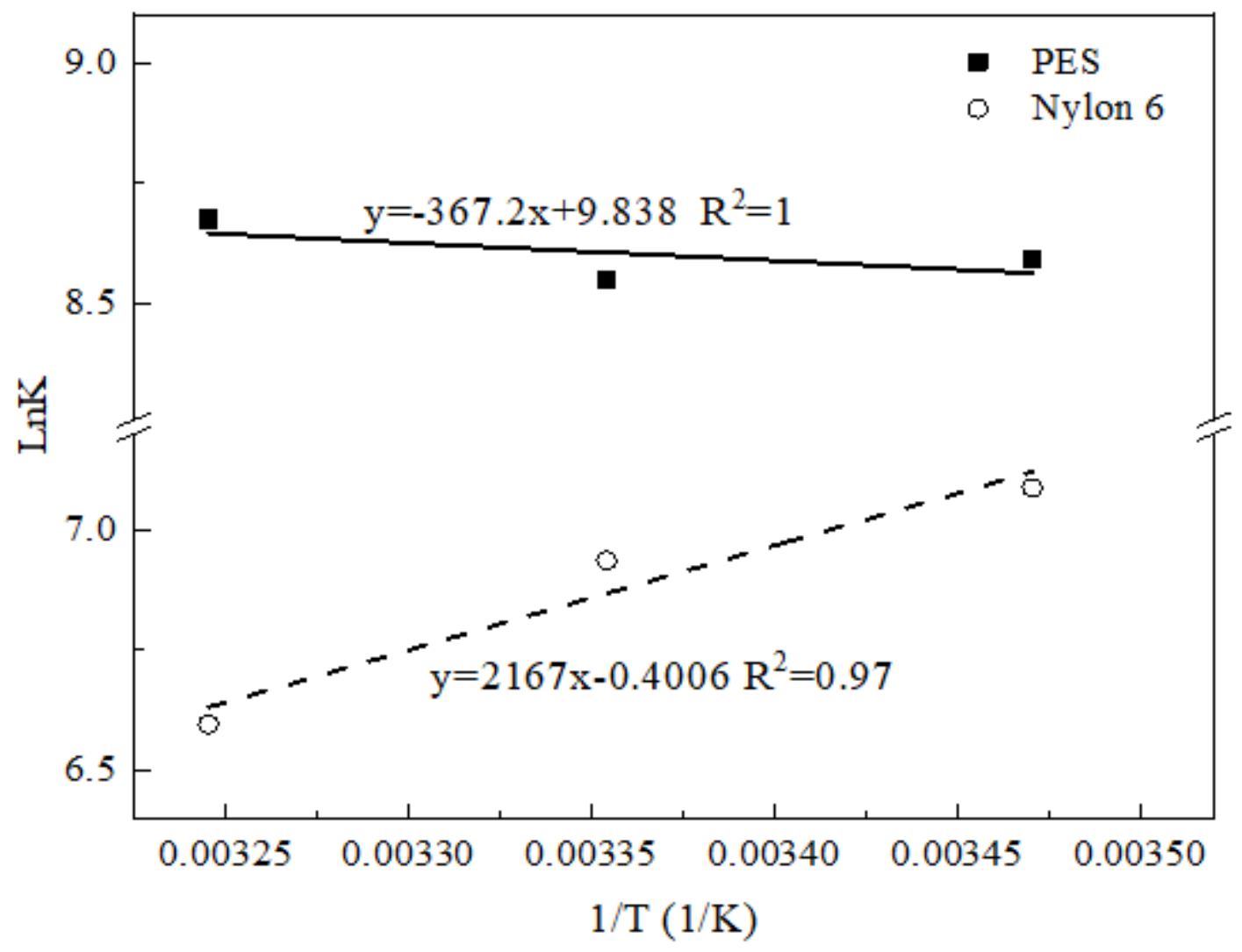

Figure 2

Plot of InK against 1/T for the estimation of thermodynamic parameters for the pyraclostrobin adsorption on PES and Nylon 6 micromembranes. 

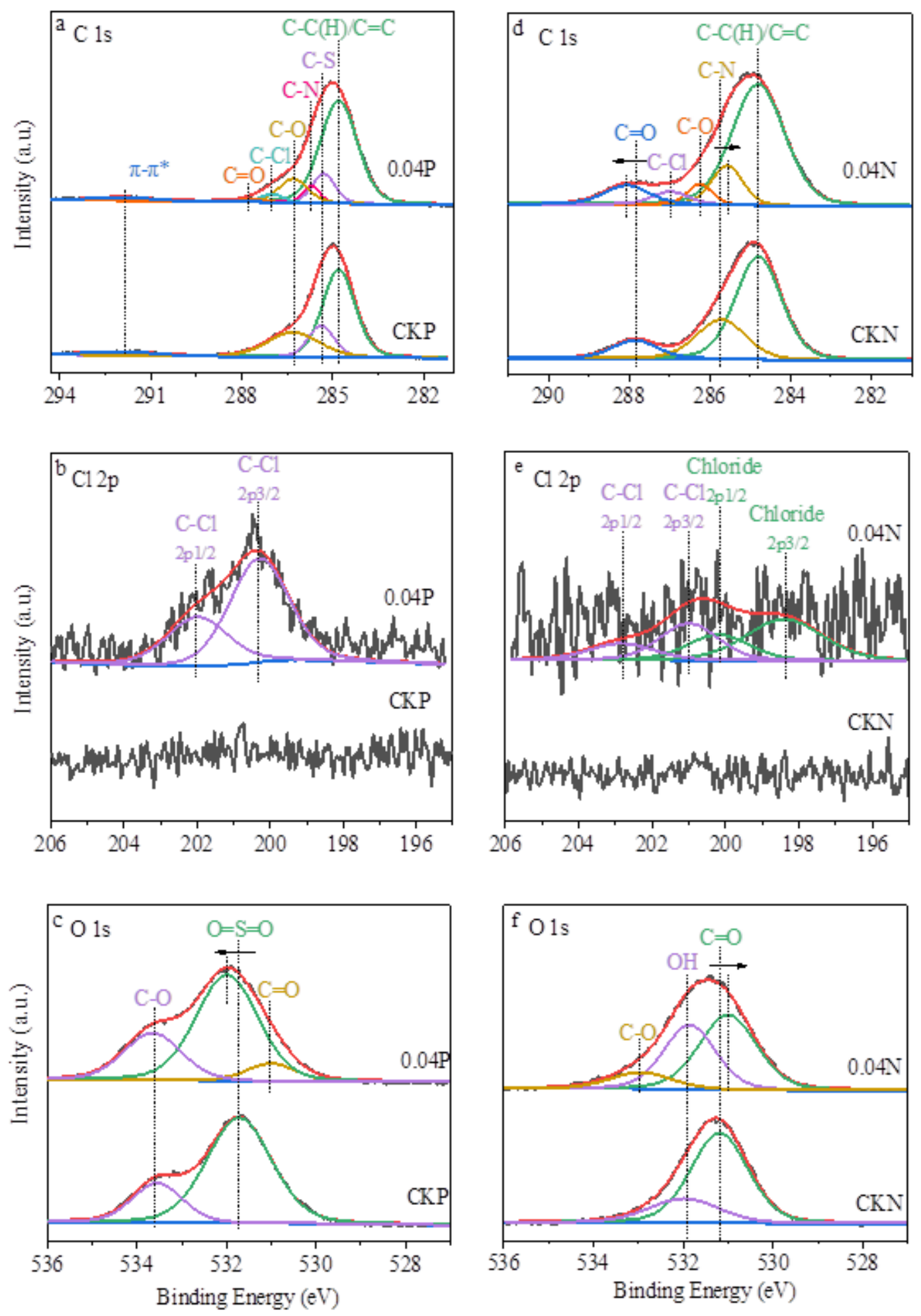

\section{Figure 3}

The $\mathrm{C} 1 \mathrm{~s}(\mathrm{a}), \mathrm{Cl} 2 \mathrm{p}(\mathrm{b})$ and $01 \mathrm{~s}(\mathrm{c})$ XPS spectra of PES micromembrane before (CKP) and after (0.04P) pyraclostrobin adsorption.The $\mathrm{C} 1 \mathrm{~s}(\mathrm{~d}), \mathrm{Cl} 2 \mathrm{p}(\mathrm{e})$ and $01 \mathrm{~s}(\mathrm{f})$ XPS spectra of Nylon6 micromembranes before $(\mathrm{CKN})$ and after $(0.04 \mathrm{~N})$ pyraclostrobin adsorption. 
(a)

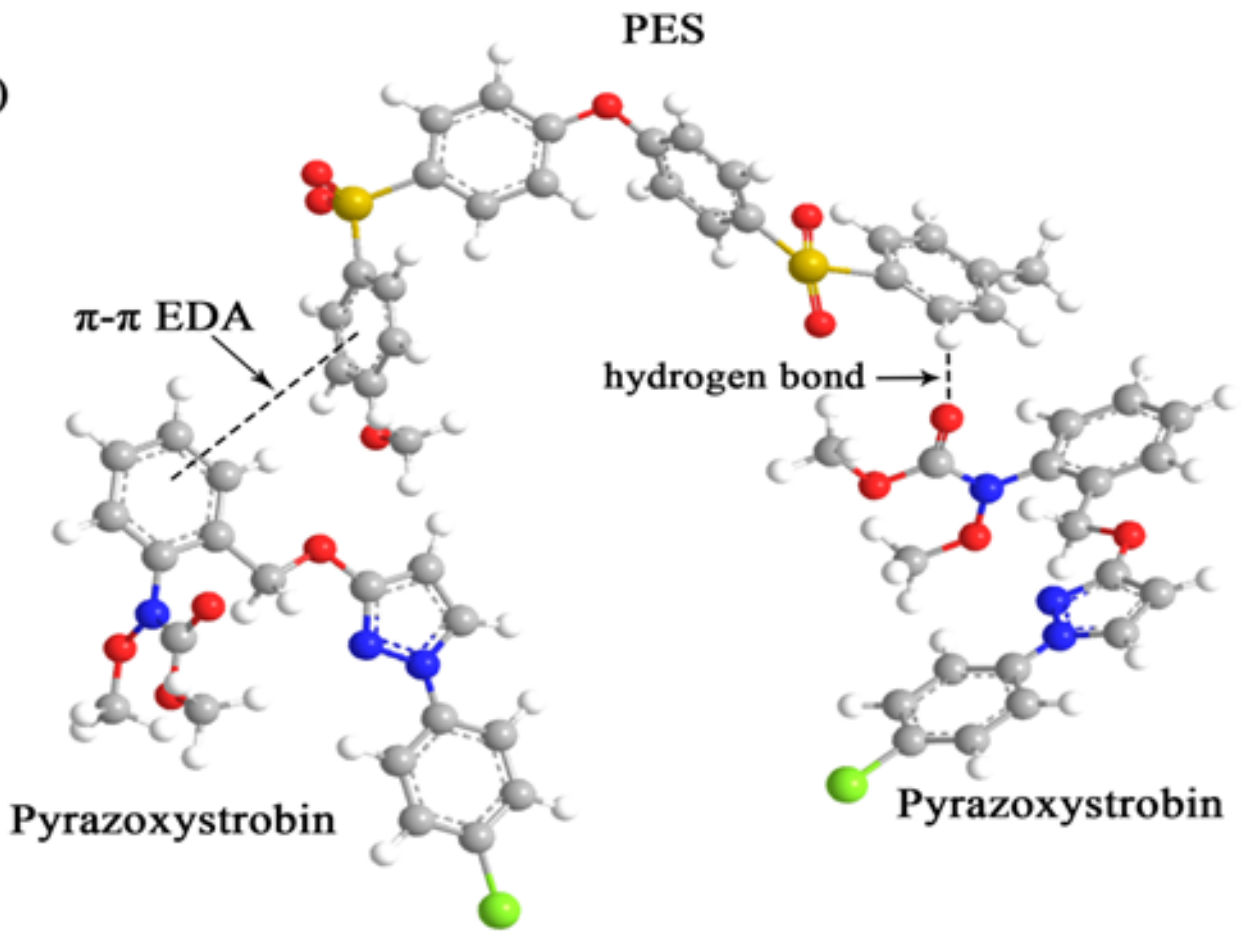

(b)

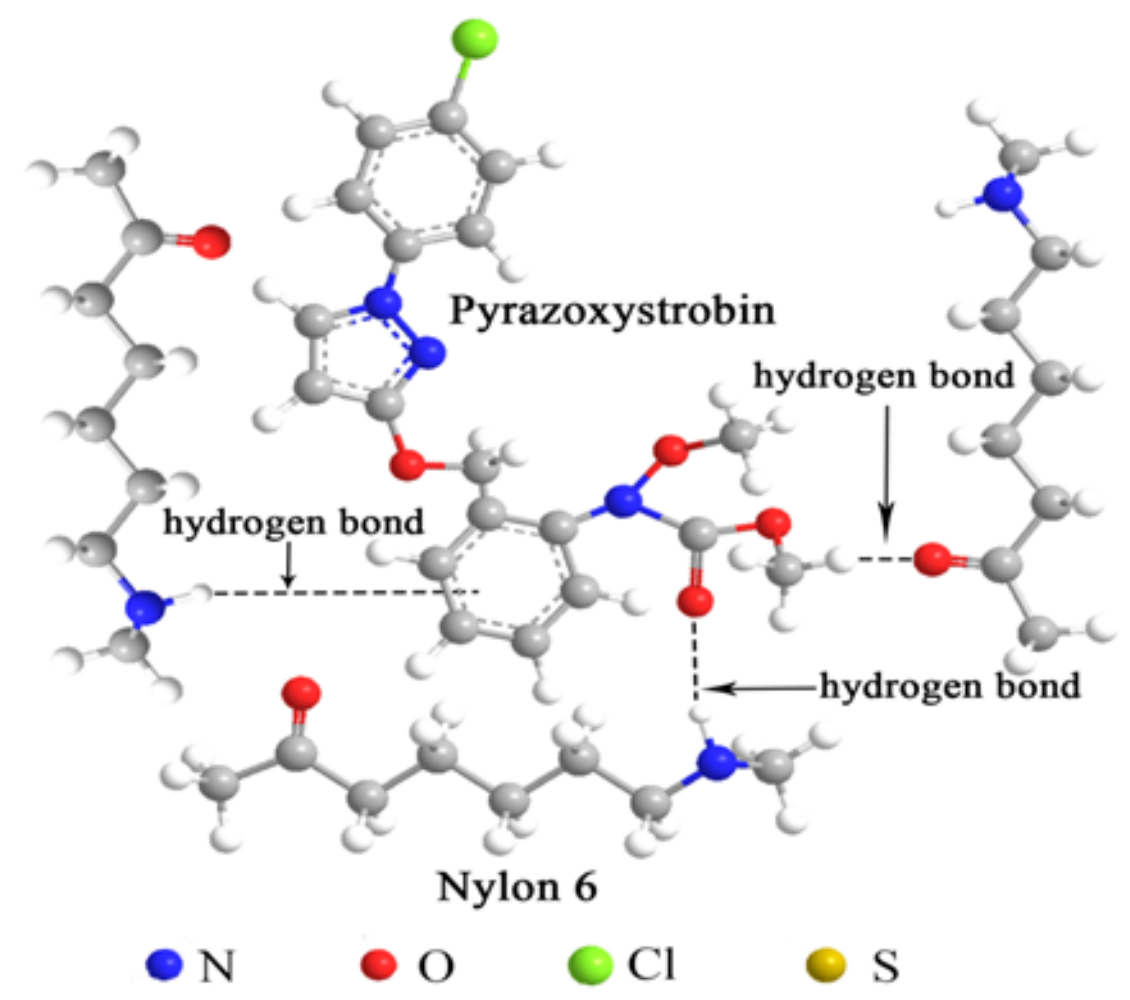

Figure 4

Schematic diagram of the adsorption mechanisms of pyraclostrobin by Polyethersulfone (a) and Nylon 6 micromembrane (b). 

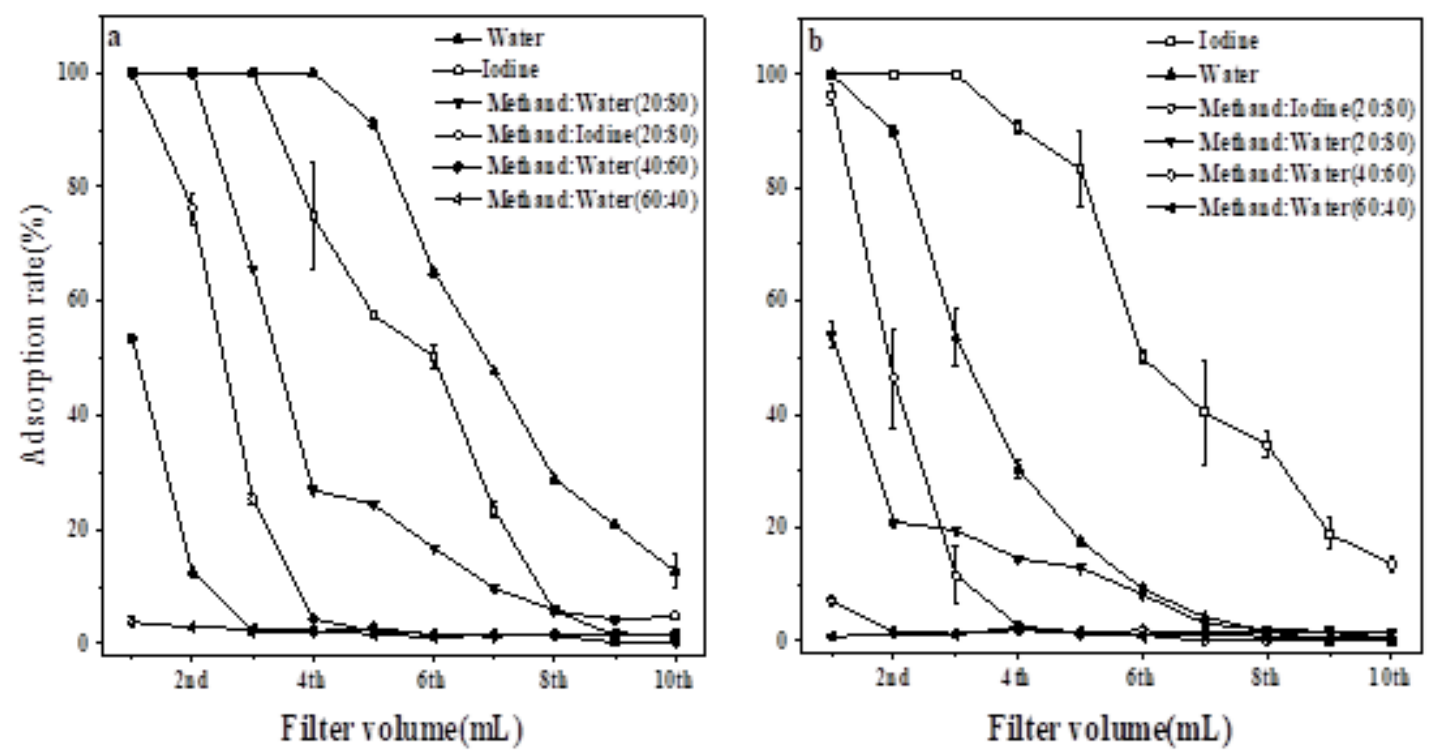

Figure 5

The effect of methanol and iodine on the adsorption of PES (a) and Nylon6 (b) micromembrane filters to pyraclostrobin. $(\mathrm{pH}=6.92$, temperature $=298.13 \mathrm{~K}$, error bars represent standard deviation from the mean $(n=3))$.

\section{Supplementary Files}

This is a list of supplementary files associated with this preprint. Click to download.

- GraphicalAbstract.png

- Supplementaryfile..docx 